\title{
Study of the Nonparametric Kaplan-Meier Estimator of the Cumulative Incidence Function in Competiting Risks
}

\author{
Didier Alain Njamen Njomen ${ }^{1}$ \\ ${ }^{1}$ Department of Mathematics and Computer Science, Faculty of Science, University of Maroua, Cameroon \\ Email: didiernjamen1@gmail.com and didier.njamen@univ-maroua.cm
}

\begin{abstract}
In this paper, inspired by the estimator of the cumulated specific incidence proposed by Marubini and Vasecchi [23], we obtain the Kaplan-Meier estimator of the survival function of all causes of death combined by summing the estimator of $f_{j}(t)(j \in\{1, \ldots, m\})$ obtained by plug-in. We establish that the Kaplan-Meier estimator of the survival function overestimates the cumulative incidence in the presence of competitive events. By making the product of all the contributions of the studied system, we establish the likelihood function of the specific risk function for the competitive risk model. Finally, under the assumptions of Dinse and Larson [13], and using the delta-method, we establish the variance of the cumulative incidence function in competiting risks.
\end{abstract}

Keywords: Survival analysis; competiting risks; function of cumulative incidence; Kaplan-Meier estimators; risk function of specific cause; likelihood function; delta-method.

MSC 2000: 62Nxx, 62NO1, 62-07, 62Gxx, 62NO2, 62G05.62Hxx, 62J10

\section{Introduction}

\subsection{Concept of Survival Time Analysis}

For decades, many statisticians have always sought to model the distribution of a random variable $T$ representing a survival time. The interested reader will be able to consult Aalen [1], [2], Kalbfleisch and Prentice [19], Cox and Oakes [12], Miller [24], Carbon and al. [8], Fleming and Harrington [14], Andersen and al. [3], [4], [5], Hill and al. [17], Klein and Moeschberger [21], Saranya and Karthikeyan [29] to name but a few.

The term "death" will be synonymous thereafter occurrence of the event of interest.

The law of $T$ is uniquely determined by its distribution function:

$$
F(t)=\mathbb{P}(T \leq t), \quad t \geq 0 .
$$

$F(t)$ is also called cumulative incidence function. For $t$ fixed, it represents the probability of dying before the instant $t$. Equivalently with the other probability laws, we can deduce from the cumulative incidence function a density function such that:

$$
f(t)=F(t)^{\prime}=\lim _{h \rightarrow 0}\left(\frac{F(t+h)-F(t)}{h}\right) .
$$

The area under the curve between two points of the abscissa axis $t_{1}$ and $t_{2}$ results in the probability of dying between these two instants.

We also introduce a function directly calculated from those mentioned above, which models the probability of dying at the instant $t$ for a patient knowing that this patient has "survived" up to the instant $t$ ("To survive" means not to die in the sense described above). This is the instant risk function defined by:

$$
\lambda(t)=\mathbb{P}(T=t \mid T \geq t)=\lim _{h \rightarrow 0}\left(\frac{\mathbb{P}(t \leq T \leq t+h \mid T \geq t)}{h}\right)=\frac{f(t)}{1-F\left(t^{-}\right)} .
$$

Its cumulative version is defined by:

$$
\Lambda(t)=\int_{0}^{t} \lambda(u) d u
$$


Remark 1.1 There are five functions in survival data analysis. The interested reader may consult Huber ([18], pp. 4-5) and Njamen ([26], p. 4). These functions are strongly related to each other. Indeed:

$$
\begin{aligned}
\Lambda(t) & =\int_{0}^{t} \lambda(u) d u \\
& =\int_{0}^{t} \frac{f(u)}{1-F\left(u^{-}\right)} d u \\
& =\int_{0}^{t} \frac{-\left(1-F\left(u^{-}\right)\right)^{\prime}}{1-F\left(u^{-}\right)} d u \\
& =-[\ln (1-F(u))]_{0}^{t} \\
& =-\ln (1-F(t)) \\
& \Leftrightarrow 1-F(t)=\exp (-\Lambda(t)) .
\end{aligned}
$$

The function of total survival i.e. the probability of not yet down at time $t$, can be written as follows :

$$
S(t)=\mathbb{P}(T>t)=e^{\left\{-\int_{0}^{t} \lambda(u) d u\right\}} .
$$

\subsection{Censor}

In reality, not all the data collected are realizations of the $T$ random variable. Indeed, the observation may stop while no deaths have been observed for some patients. Nevertheless the observation period is postponed, since it contains the following information: "the patient $i$ did not die during the observation period", it's about a censored patient.

The following notations are thus introduced to translate this type of observation (called right random censorship to signify that the observations have been censored on the right): we actually observe the random variable $Z=\min (T, C)$ representing the duration until the occurrence of the first event among two possibilities: the death and censorship. The random variable $T$ measures the time until death and the variable $C$ measures the time until censorship. To indicate which event occurred, we also introduce the censoring indicator $\delta=\mathbb{1}_{\{T \leq C\}}$ which is 0 in case of censorship, and 1 if the duration of interest is observed.

Finally, the data for the patient $i$ are in the form of the triplets $\left(z_{i}, \delta_{i}, y_{i}\right)$ realization of the random vector $(Z, \Delta, Y)$ where $z$ represents the observed duration, $\delta$ the censored binary variable and $y$ the vector containing the patient's personal data, called covariates or exogenous variables.

\section{Estimation of Nonparametric Estimators of Nelson-Aalen and Kaplan-Meier in the Presence of Right Random Censorship}

Nelson-Aalen's estimator of the cumulative risk $\Lambda(t)$ in the presence of right random censorship is defined by:

$$
\widehat{\Lambda}_{n}(t)=\sum_{i^{\prime}=1, T_{i}^{\prime} \leq t}^{n^{\prime}} \frac{M_{i^{\prime}}}{R_{i^{\prime}}}
$$

where $M_{i^{\prime}}$ is the number of deaths observed in $t_{i^{\prime}}, R_{i^{\prime}}$ the number of subjects neither dead nor censored just before $t_{i^{\prime}}$ (subjects said "to risks "), and $T^{\prime}$ is the increasing ordered version of $T$, which is the random variable representing the time until the event of interest for uncensored patients. It is wise to recall that $i=1, \ldots, n$ represents the observed individuals and $i^{\prime}=1, \ldots, n^{\prime}$ the observed and uncensored individuals sorted in ascending order of observed time (so, we have $n^{\prime} \leq n$ ). 
The Kaplan-Meier estimator of cumulated incidence in the presence of right random censorship is defined by:

$$
1-\widehat{F}_{n}(t)=\sum_{i^{\prime}=1, T_{i}^{\prime} \leq t}^{n^{\prime}}\left(1-\frac{M_{i^{\prime}}}{R_{i^{\prime}}}\right)=\widehat{S}_{n}(t) .
$$

\section{Competiting Risk Model}

\subsection{Introduction and Definition}

In lifetimes, we usually look at the time of death of an individual or the downtime of a system in industrial reliability. In some experiments, there may be several possible causes of death or breakdown. This is called competiting risk. Competiting events are the events that cause the observations to stop (except for the event of interest). In the theory, these events are considered as integral part of right random censorship. We have seen that, to provide the estimates of the various functions above, it must be assumed that this censorship is independent of the event of interest. This assumption is not valid in all cases (see Tsiatis [31] and Prentice [28]). The interested reader will be able to consult Gray [15], Heckman and Honoré [16], Commenges [10], Com-nougué [11], Crowder [9], Peña and al. [27], Klein and Moeschberger [21], Latouche [22], Klein [20], Belot [6], Stocker IV and Adekpedjou [30], Njamen and Ngatchou [25], Njamen [26], Martinez-Camblor [7], etc.

It is then necessary to modify the modeling of the data.

\subsection{Modeling the Model in Competiting Risks}

For each system, we observe $T=\min \left(\tau_{1}, \ldots, \tau_{m}\right)$, i.e. the time the system goes down due to causes $m$. Moreover, we denote by $\eta$ the cause of failure, i.e. $\eta=j$ if $T=\tau_{j}$ for all $j=1, \ldots, m$. This implies that $\tau_{1}, \ldots, \tau_{m}$ are latent random variables, i.e. they are not observable. Indeed, only the variables $T$ and $\eta$ are observed.

In the theory, we observe the pairs $\left(z_{i}, j_{i}\right)$ which represent the subject $i$ where $z$ is a realization of $Z$ (time to the first event among the $m+1$ possible with censorship ) and $j$ realization of $\eta$ random variable representing the first event undergone (among the $m+1$ possible events).

This modeling was used in Latouche's ([22], p.8), Belot's ([6], p.12), and in Njamen and Ngatchou $([25]$, p.5).

New specific functions are directly drawn from this modeling:

\subsection{Estimation of Distribution Functions for Specific Causes}

3.3.1 Cumulative Incidence Function for Specific Causes The law of the pair $(T, \eta)$ is entirely determined by the set of cumulative incidence (cumulative incidence function) functions:

$$
F_{j}(t)=\mathbb{P}(T \leq t \cap \eta=j), \quad j \in\{1, \ldots, m\} .
$$

These are distribution subfunctions, i.e. $F_{j}(t)$ is not a true function. Indeed:

- $\lim _{t \rightarrow \infty} F_{j}(t)=\mathbb{P}(\eta=j) \leq 1$;

- $\sum_{j=1}^{m} F_{j}(t)=\mathbb{P}(T \leq t)=F(t)$, the distribution function of $T$ presented in (1).

However, this function is non-decreasing with $F_{j}(0)=0$ and $F_{j}(\infty) \leq 1$.

Equation (8) represents the "probability" of break down the caused by $j$ before the time $t$. 
3.3.2 Density Function with Specific Causes Let $f_{j}(t)$ be the ("sub")-density associated with $F_{j}(t)$ such that:

$$
f_{j}(t)=F_{j}(t)^{\prime}=\lim _{h \rightarrow 0}\left(\frac{F_{j}(t+h)-F_{j}(t)}{h}\right)=\lim _{h \rightarrow 0}\left(\frac{\mathbb{P}((t \leq T \leq t+h) \cap \eta=j)}{h}\right) .
$$

In the same way that $F_{j}(t)$, we have $\sum_{j=1}^{m} f_{j}(t)=f(t)$, the density associated with $T$ presented in (2).

3.3.3 Risk Function Specific to Specific Causes The specific risk function is the instant probability of dying in $t$ of cause $j$ knowing that one has survived all causes up to $t$.

$$
\begin{aligned}
\lambda_{j}(t) & =\lim _{h \rightarrow 0}\left(\frac{\mathbb{P}((t \leq T \leq t+h) \cap \eta=j \mid T \geq t)}{h}\right) \\
& =\lim _{h \rightarrow 0}\left(\frac{\mathbb{P}((t \leq T \leq t+h) \cap \eta=j \mid T \geq t)}{\mathbb{P}(T \geq t)}\right) \times \frac{1}{h} \\
& =\frac{f_{j}(t)}{1-F\left(t^{-}\right)} .
\end{aligned}
$$

The total risk function, i.e. the risk function for all causes of failure combined, is simply given by

$$
\lambda(t)=\sum_{i=1}^{m} \lambda_{j}(t)
$$

$\lambda(t)$ represents the rate at which the systems which have not yet fail of none of $m$ causes at $t$ time break down any cause.

The density function with specific causes defined in (9) can now be expressed in terms of $\lambda_{j}$ and $\lambda$ for all $j \in\{1, \ldots, m\}$ by:

$$
f_{j}(t)=\lambda_{j}(t) e^{\left\{-\int_{0}^{t} \lambda(u) d u\right\}}
$$

3.3.4 Survival Function for Specific Causes The survival function for specific causes is defined by:

$$
S_{j}(t)=e^{\left\{-\int_{0}^{t} \lambda_{j}(u) d u\right\}}, \quad j \in 1, \ldots, m .
$$

The total survival function defined in (5) which is the probability of not yet have brokendown of none of $m$ cause at $t$ time, can be expressed in the following way:

$$
S(t)=\prod_{j=1}^{m} S_{j}(t)
$$

Indeed:

$$
\begin{array}{rlrl}
S(t) & =e^{\left\{-\int_{0}^{t} \lambda(u) d u\right\}} & & \text { according (8) } \\
& =e^{\left\{-\int_{0}^{t}\left[\lambda_{1}(u), \ldots, \lambda_{m}(u)\right]\right\}} & \text { according (7) } \\
& =e^{\left\{-\int_{0}^{t} \lambda_{1}(u)\right\}} \ldots e^{\left\{-\int_{0}^{t} \lambda_{m}(u)\right\}} & \\
& =S_{1}(t) \ldots S_{m}(t) & & \text { according (8) } \\
& =\prod_{j=1}^{m} S_{j}(t) . &
\end{array}
$$

If the competiting risks $\tau_{1}, \ldots, \tau_{m}$ are not independent, then $S_{j}(t), j=1, \ldots, m$ can not be interpreted as a probability or a function of survival. On the other hand, if the competitive risks $\tau_{1}, \ldots, \tau_{m}$ are independent, then $S_{j}(t)$ is the marginal survival function of $\tau_{j}$, i.e. it can be seen as the probability of surviving at least until $t$, in an environment where the only possible cause of failure is $j$. 
3.3.5 Specific Risk Function Cumulated with Specific Causes The cumulative specific risk function is deduced from relation (10).

$$
\Lambda_{j}(t)=\int_{0}^{t} \lambda_{j}(u) d u=-\ln \left(S_{j}(t)\right), \quad j \in\{1, \ldots, m\},
$$

which is worth $+\infty$ when $S_{j}(t)=0$.

Remark 3.1 It is interesting to remember that relation (14) is valid only when $F$ is continuous. When $F$ is arbitrary, the cumulative risk function is

$$
\Lambda_{j}(t)=\int_{0}^{t} \frac{d F_{j}(s)}{1-F(s)} .
$$

3.3.6 Application of the Estimation Method in Competiting Risks to the Usual Laws The estimation method can be applied to specific causes to particular distributions. Knowing the survival function and the risk function of the law in question, we can proceed to the different calculations: (in this paper, we treat only the case of Weibull's law) : for the case of Weibull's law, the functions of survival, cumulative risk and risk of the cause $j$ are respectively given by:

$$
\begin{aligned}
& S_{j}(t)=e^{\left\{-\left(\frac{t}{\theta_{j}}\right)^{\delta_{j}}\right\},}, j \in\{1, \ldots, m\} ; \\
& \Lambda_{j}(t)=\left(\frac{t}{\theta_{j}}\right)^{\delta_{j}}, \quad j \in\{1, \ldots, m\} ; \\
& \lambda_{j}(t)=\frac{\delta_{j}}{\theta_{j}}\left(\frac{t}{\theta_{j}}\right)^{\delta_{j}-1}, j \in\{1, \ldots, m\} .
\end{aligned}
$$

\section{Insufficiency of Functions Related to Specific Causes}

\subsection{Risk Function of Gray ([15])}

The functions described in Section 3 are not satisfactory for interpreting the results correctly. This is why Gray ([15]) intuitively introduces the risk function associated with the cumulative incidence function of cause $j$ defined by:

$$
\gamma_{j}(t)=\frac{f_{j}(t)}{1-F_{j}\left(t^{-}\right)}=-\frac{\delta}{\delta t} \ln \left(1-F_{j}(t)\right) .
$$

The development of this quantity allows us to notice certain irregularities. By replacing $f_{j}(t)$ and $F_{j}(t)$ respectively by their definitions, we have:

$$
\begin{aligned}
\gamma_{j}(t) & =\lim _{h \rightarrow 0}\left(\frac{\frac{\mathbb{P}((t \leq T \leq t+h) \cap \eta=j)}{h}}{1-\mathbb{P}(T<t \cap \eta=j)}\right) \\
& =\lim _{h \rightarrow 0}\left(\frac{\mathbb{P}(((t \leq T \leq t+h) \cap \eta=j) \cap(T \geq t \cup(T \leq t \cap \eta \neq j)))}{h \times \mathbb{P}(T \geq t \cup(T \leq t \cap \eta \neq j))}\right) \\
& =\lim _{h \rightarrow 0}\left(\frac{\mathbb{P}((t \leq T \leq t+h) \cap \eta=j \mid(T \geq t \cup(T \leq t \cap \eta \neq j)))}{h}\right) .
\end{aligned}
$$

To obtain (17), we have remarked that:

$$
\begin{aligned}
1-\mathbb{P}(T<t \cap \eta=j) & =\mathbb{P}(T \geq t \cup \eta \neq j) \\
& =\mathbb{P}(T \geq t \cup((T>t \cup T \leq t) \cap \eta \neq j)) \\
& =\mathbb{P}(T \geq t \cup((T>t \cap \eta \neq j) \cup(T \leq t \cap \eta \neq j))) \\
& =\mathbb{P}((T \geq t \cup(T>t \cap \eta \neq j)) \cup(T \leq t \cap \eta \neq j)) \\
& =P(T \geq t \cup(T \leq t \cap \eta \neq j)),
\end{aligned}
$$


and that :

$$
\begin{aligned}
\mathbb{P}((t & <T \leq t+h) \cap \eta=j) \cap(T>t \cup(T \leq t \cap \eta \neq j))) \\
& =\mathbb{P}(((t<T \leq t+h) \cap \eta=j) \cap(T>t)) \cup(((t<T \leq t+h) \cap \eta=j) \cap(T \leq t \cap \eta \neq j))) \\
& =\mathbb{P}(((t<T \leq t+h) \cap \eta=j) \cup \emptyset) \\
& =\mathbb{P}((t<T \leq t+h) \cap \eta=j) .
\end{aligned}
$$

It will be noted that the function found above corresponds to the instant risk function of the random variable (improper) $T_{j}^{*}=\mathbb{1}_{\{\eta=j\}} T+\mathbb{1}_{\{\eta \neq j\}} \infty$. Indeed :

$$
\begin{aligned}
\lambda_{T_{j}^{*}}(t) & =\lim _{h \rightarrow 0} \frac{\mathbb{P}\left(t \leq T_{j}^{*} \leq t+h \mid T_{j}^{*} \geq t\right)}{h} \\
& =\lim _{h \rightarrow 0} \frac{\mathbb{P}\left(t \leq\left(\mathbb{1}_{\{\eta=j\}} T+\mathbb{1}_{\{\eta \neq j\}} \infty\right) \leq t+h \mid\left(\mathbb{1}_{\{\eta=j\}} T+\mathbb{1}_{\{\eta \neq j\}} \infty\right) \geq t\right)}{h} \\
& =\lim _{h \rightarrow 0} \frac{\mathbb{P}([t \leq T \leq t+h) \cap(\eta=j)] \cup[(t \leq \infty \leq t+h) \cap(\eta \neq j)] \mid(T \geq t \cap \eta=j) \cup(\infty \geq t \cap \eta \neq j))}{h} \\
& =\lim _{h \rightarrow 0} \frac{\mathbb{P}([t \leq T \leq t+h) \cap(\eta=j)] \cup[\emptyset \cap(\eta \neq j)] \mid(T \geq t \cap \eta=j) \cup(\Omega \cap \eta \neq j))}{h}
\end{aligned}
$$

(because $t, t+h<\infty$, so the set $\{t<\infty<t+h\}$ is the empty set and the set $\infty>t$ is the $\Omega$ universe)

$$
\begin{aligned}
& =\lim _{h \rightarrow 0} \frac{\mathbb{P}((t \leq T \leq t+h) \cap(\eta=j) \mid(T \geq t \cap \eta=j) \cup(\eta \neq j))}{h} \\
& =\lim _{h \rightarrow 0} \frac{\mathbb{P}((t \leq T \leq t+h) \cap(\eta=j) \mid((T \geq t) \cup(\eta \neq j)) \cap(\eta=j \cup \eta \neq j))))}{h} \\
& =\lim _{h \rightarrow 0} \frac{\mathbb{P}((t \leq T \leq t+h) \cap(\eta=j) \mid(T \geq t) \cup(\eta \neq j)))}{h} . \\
& =\gamma_{j}(t)
\end{aligned}
$$

The preceding calculations prove (see relations (19) and (20)) that the instant risk function of the random variable (improper)

$$
T_{j}^{*}=\mathbb{1}_{\{\eta=j\}} T+\mathbb{1}_{\{\eta \neq j\}} \infty,
$$

is equal to the function of Gray $([15])$, which is not the case in the classical framework.

This intuitive remark suggests a new transformation of our data: the measured time becomes $T$ if $\eta=1$ (the event of interest) and $\infty$ otherwise.

\subsection{New Modeling Adapted to the Risk Function of Gray ([15])}

The support for right random censorship (constituted of events for which the independence assumption is likely) is similar to that previously presented in subsection 3.2 . We pose :

$$
Z=\min \left(T_{j}, T_{1} \ldots T_{j-1}, T_{j+1} \ldots T_{m}, C\right),
$$

where $T_{j}$ is the duration until the event of interest (here the jth), $T_{k}$ with $k=1 \ldots j-1, j+1 \ldots m$ is the duration until the competitive event $k$, and $C$ is the measured duration until censorship.

We pose respectively

$$
\eta=k \text { if } \min \left(T_{j}, T_{1} \ldots T_{j-1}, T_{j+1} \ldots T_{m}, C\right)=T_{k},
$$

and

$$
\Delta=0 \text { if } \min \left(T_{j}, T_{1} \ldots T_{j-1}, T_{j+1} \ldots T_{m}, C\right)=C \text { and } \Delta=1 \text { if not. }
$$


So, for technical reasons, we consider the random variable $\xi=\eta \delta$ such that $\xi=j$ if $(T \leq C$ and $\eta=j)$ and $\xi=0$ if $T>C$. We note that $\delta$ and $\xi$ are observable and $\eta$ is so only for $T$ uncensored.

In reality, in practice, we observe the triplets $\left(z_{i}, \xi_{i}, y_{i}\right)$ realization of the random variables $(Z, \Delta * \eta, Y)$, where

$$
\xi=\delta * \eta=\left\{\begin{array}{l}
0 \\
j
\end{array}\right.
$$

and

$$
Z_{i}=\min \left(T_{i}, C_{i}\right), \quad \delta_{i}=\mathbb{1}_{\left\{T_{i} \leq C_{i}\right\}}, \quad \text { with } T_{i}=\min \left(\tau_{1}^{i}, \ldots, \tau_{m}^{i}\right)
$$

and where $\tau_{j}^{i}$ is the time that an individual $i$ is subject to the cause $j$. This technique has been used in Njamen and Ngatchou ([25], p.5) for the evaluation of the specific functions of distribution to specific causes.

\section{Expression of the Kaplan-Meier Estimator of the Specific Cumulated Incidence in the Presence of Right Random Censorship}

In this section, we use the estimator of the specific cumulated incidence proposed by Marubini and Vasecchi ([23], p. 338).

The Kaplan-Meier estimator is thus obtained by summing the $f_{j}(t)$ estimator obtained by plug-in in $f_{j}(t)=\lambda_{j}(t) \times(1-F(t))(j=1, \ldots, m)$, and is written

$$
\widehat{F}_{j}(t)=\sum_{t \leq T_{i}^{\prime}, T_{i}^{\prime} \leq t}\left(1-\widehat{F}_{n}(t)\right) \times \frac{M_{i^{\prime} j}}{R_{i}^{\prime}}, \quad j \in\{1, \ldots, m\},
$$

so

$$
1-\widehat{F}_{n}(t)=\sum_{i^{\prime}=1, T_{i}^{\prime} \leq t}\left(1-\frac{M_{i}^{\prime}}{R_{i}^{\prime}}\right)=\widehat{S}_{n}(t)
$$

where

- $1-\widehat{F}_{n}(t)$ is the Kaplan-Meier estimator for all causes of death combined (with $M_{i^{\prime}}=\sum_{j} M_{i^{\prime} j}$ ) and

- $\frac{M_{i^{\prime} j}}{R_{i}^{\prime}}$ the estimator of $\lambda_{j}\left(t_{i^{\prime}}\right)$ with $M_{i^{\prime} j}$ the number of deaths caused by $j$ in $T_{i}^{\prime}$ and

- $R_{i^{\prime}}$ the number of subjects at risk in $T_{i}^{\prime}$, that is to say, neither censored nor died in any other cause than $j$.

These different additional information provided by the Kaplan-Meier estimator obtained by summing the estimator of $f_{j}(t)$ obtained by plug-in in $f_{j}(t)=\lambda_{j}(t) \times(1-F(t))(j=1, \ldots, m)$ allow us to achieve the following results:

\section{Main Results}

\subsection{Overestimate of Estimate of Cumulative Incidence}

From the writing of this estimator (see above), we note that the Kaplan-Meier estimator estimates a function that is not the specific cumulated incidence sought. Thus, the following result constitutes the first of this paper.

Proposition 6.1 In the presence of competitive events, the Kaplan-Meier estimator overestimates the cumulative incidence. 
Proof If we are interested in the cause of death 1 (chosen from the $m$ possible) we have:

$$
F_{1}(t)=\int_{0}^{t} S\left(u^{-}\right) \lambda_{1}(u) d u \text { is estimated by (22) with } j=1 .
$$

On the other hand,

$$
\begin{aligned}
S(u)=e^{(-\Lambda(t))} & =e^{\left(-\left(\sum_{j=1}^{m} \Lambda_{j}(t)\right)\right)} \\
& =\int_{0}^{t} e^{\left(-\left(\sum_{j=1}^{m} \Lambda_{j}\left(u^{-}\right) \lambda_{1}(u) d u\right)\right)} \\
& =\int_{0}^{t} e^{\left(-\left(\sum_{j=1, j \neq 1}^{m} \Lambda_{j}\left(u^{-}\right)\right)\right)} e^{\left(-\lambda_{1}(u)\right)} \lambda_{1}(u) d u .
\end{aligned}
$$

We pose

$$
e^{\left(-\left(\sum_{j=1, j \neq 1}^{m}\right)\right)}=C(u)
$$

and we notice that

$$
C(u) \leq 1
$$

If we now consider the $m-1$ other competitive events as non-informative censorship, we assume that there is only one cause of death, the 1 . So,

$$
S^{\prime}(u)=e^{\left(-\left(\sum_{j=1}^{1} \Lambda_{j}(t)\right)\right)}=e^{\left(-\Lambda_{1}(t)\right)} .
$$

In this particular case, we have :

$$
\begin{aligned}
F^{\prime}(t) & =\int_{0}^{t} S\left(u^{-}\right) \lambda_{1}(u) d u \\
& =\int_{0}^{t} e^{\left(-\Lambda_{1}\right)} \lambda_{1}(u) d u \text { estimated by } 1-\widehat{S}(t) \text { where } \widehat{S}(t) \text { is, the estimator of Kaplan }- \text { Meier } \\
& \geq \int_{0}^{t} C\left(u^{-}\right) e^{\left(-\Lambda_{j}\right)} \lambda_{j}(u) d u \quad(\operatorname{car} C(u) \leq 1 \forall u) \text { estimated by }(22) .
\end{aligned}
$$

Thus, the Kaplan-Meier estimator's complement to 1 at a competiting risk event (but neglecting it) estimates a function that is not the specific cumulated incidence sought, but a function superior or equal (strictly superior so far as $\sum_{l=1, l \neq j}^{m} \Lambda_{l}\left(u^{-}\right)>0$ ), thus the overestimate.

This ends the proof of the proposition.

\subsection{Likelihood Function in Competiting Risks}

Starting from the remark that each contribution results in a system that breaks down from the cause $j_{i}(i=1, \ldots, n$ avec $j \in\{1, \ldots, m\})$ at time $\tau_{i}$ or by the censoring the system at time $\tau_{i}$ and on the other hand, by making the product of all the contributions of the system $i$, one succeeds in establishing the likelihood function of the specific risk function related to the specific causes, hence, the second fundamental result of this paper. 
Theorem 6.1 In an $n$ system where we observe the triple $\left(\tau_{i}, \delta_{i}, j_{i}\right)$, realization of $(Z, \Delta, \eta)$, the likelihood function denoted $L\left(\lambda_{j}\right)$ for the competiting risk model is given by:

$$
L\left(\lambda_{j}\right)=\prod_{i=1}^{n}\left(\left\{\lambda_{j_{i}}\left(\tau_{i}\right)\right\}^{\delta_{i}} \prod_{j=1}^{m} e^{-\int_{0}^{t} \lambda_{j}(u) d u}\right)
$$

where

- $\tau_{i}$ is the time of failure or censorship observed for the system $i$;

- $\delta_{i}$ is the indicator that is 1 if the system $i$ has a failure, and 0 otherwise;

- $j_{i}$ is the cause of system $i$ failure.

Proof For $i=1, \ldots, n$, the product of the $i$ system contribution is translated by:

$$
\begin{aligned}
L\left(\lambda_{j}\right) & =\prod_{i=1}^{n}\left\{\left(f_{j_{i}}\left(\tau_{i}\right)\right)^{\delta_{i}}\left(S\left(\tau_{i}\right)\right)^{1-\delta_{i}}\right\} \\
& =\prod_{i=1}^{n}\left\{\left(\lambda_{j_{i}}\left(\tau_{i}\right) S\left(\tau_{i}\right)\right)^{\delta_{i}}\left(S\left(\tau_{i}\right)\right)^{1-\delta_{i}}\right\} \text { from (**) } \\
& =\prod_{i=1}^{n}\left\{\left(\lambda_{j_{i}}\left(\tau_{i}\right)\right)^{\delta_{i}} S\left(\tau_{i}\right)\right\} \\
& =\prod_{i=1}^{n}\left\{\left(\lambda_{j_{i}}\left(\tau_{i}\right)\right)^{\delta_{i}} \prod_{j=1}^{m} S_{j}\left(\tau_{i}\right)\right\} \\
& =\prod_{i=1}^{n}\left\{\left(\lambda_{j_{i}}\left(\tau_{i}\right)\right)^{\delta_{i}} \prod_{j=1}^{m} e^{-\int_{0}^{t} \lambda_{j}(u) d u}\right\} \text { from } \quad \text { from }(13)
\end{aligned}
$$

This ends the proof of the theorem.

To observe a time of censorship for the system $i$ at time $\tau_{i}$, it would be necessary, for example, that this system did not always have broken down at the end of the study. In this case, we say that the system $i$ is censored to the right at $\tau_{i}=\tau$, that is to say that its downtime is greater than the end time of the study $\tau$

\subsection{Variance of Cumulated Incidence Function in the Presence of Competiting Risks}

Taking inspiration from section 5 , we have:

$$
\widehat{F}_{j}(t)=\sum_{t \leq T_{i}^{\prime}}\left(\widehat{S}(t) \times \widehat{\lambda}_{j}(t)\right), \quad j \in\{1, \ldots, m\},
$$

and this expression allows us to directly write the variance of $\widehat{F}_{j}(t)$ denoted $\operatorname{Var}\left(\widehat{F}_{j}(t)\right)$ and defined by:

$$
\operatorname{Var}\left(\widehat{F}_{j}(t)\right)=\operatorname{Var}\left(\sum_{t \leq t_{i}^{\prime}}\left(\widehat{S}(t) \times \widehat{\lambda}_{j}(t)\right)\right), \quad j \in\{1, \ldots, m\}
$$

It is necessary to remember that the $T_{i}^{\prime}$ are moments of reordered distinct death, and that $i^{\prime}$ is the number of $T_{i}$ th instant of death, hence the new form of the following variance:

$$
\operatorname{Var}\left(\widehat{F}_{j}(t)\right)=\operatorname{Var}\left(\sum_{k \leq i^{\prime}}\left(\widehat{S}(t) \times \widehat{\lambda}_{j}(t)\right)\right), j \in\{1, \ldots, m\} .
$$

This last expression allowed us to have the third fundamental result: 
Theorem 6.2 The variance of the cumulated incidence function in competiting risks is given for all $j \in\{1, \ldots, m\}$ by:

$$
\begin{aligned}
\operatorname{Var}\left(\widehat{F}_{j}(t)\right)= & \operatorname{Var}\left(\sum_{k=1}^{i^{\prime}}\left(\widehat{S}(t) \times \widehat{\lambda}_{j}(t)\right)\right) \\
& +2 \sum_{k=1}^{i^{\prime}-1} \sum_{b=k+1}^{i^{\prime}} \operatorname{Cov}\left(\left(\left(\widehat{S}\left(t_{k}\right) \times \widehat{\lambda}_{j}\left(t_{k}\right)\right)\right),\left(\widehat{S}\left(t_{b}\right) \times \widehat{\lambda}_{j}\left(t_{b}\right)\right)\right) .
\end{aligned}
$$

\section{Proof}

we call back that $\widehat{S}\left(t_{k}\right)=\prod_{b=1}^{k-1}\left(1-\frac{M_{b}}{R_{b}}\right)$ and that $\widehat{\lambda}_{j}\left(t_{k}\right)=\frac{M_{j k}}{R_{k}}, j \in\{1, \ldots, m\}$.

The technique used in this proof is the delta-method.

Under the assumptions of Dinse and Larson [13], i.e. $M_{j k}, M_{1}, \ldots M_{k-1}$ are uncorrelated, we pose:

$$
g\left(M_{j k}, M_{1}, \ldots, M_{k-1}\right)=\prod_{b=1}^{k-1}\left(1-\frac{M_{b}}{R_{b}}\right) \times \frac{M_{j k}}{R_{k}} .
$$

Then, for all $j \in\{1, \ldots, n\}$, we have :

$$
\begin{aligned}
& \operatorname{Var}\left(g\left(M_{j k}, M_{1}, \ldots, M_{k-1}\right)\right)= \\
& \left(\begin{array}{c}
\frac{\delta g}{\delta M_{j k}} \\
\frac{\delta g}{\delta M_{1}} \\
\ldots \\
\frac{\delta g}{\delta M_{k-1}}
\end{array}\right)^{T} \times\left(\begin{array}{cccc}
\operatorname{Var}\left(M_{j k}\right) & \operatorname{Cov}\left(M_{j k}, M_{1}\right) & \ldots & \mathbb{C o v}\left(M_{j k}, M_{k-1}\right) \\
\operatorname{Cov}\left(M_{j k}, M_{1}\right) & \mathbb{V a r}\left(M_{1}\right) & \ldots & \ldots \\
\ldots & \ldots & \ldots & \mathbb{C o v}\left(M_{j k}, M_{k-1}\right) \\
\operatorname{Cov}\left(M_{j k}, M_{k-1}\right) & \ldots & \mathbb{C o v}\left(M_{j k}, M_{k-1}\right) & \operatorname{Var}\left(M_{k-1}\right)
\end{array}\right) \times\left(\begin{array}{c}
\frac{\delta g}{\delta M_{j k}} \\
\frac{\delta g}{\delta M_{1}} \\
\ldots \\
\frac{\delta g}{\delta M_{k-1}}
\end{array}\right) .
\end{aligned}
$$

Since the $M$ are all uncorrelated two by two, we have:

$$
\left(\begin{array}{c}
\frac{\delta g}{\delta M_{j k}} \\
\frac{\delta g}{\delta M_{1}} \\
\ldots \\
\frac{\delta g}{\delta M_{k-1}}
\end{array}\right)^{T} \times\left(\begin{array}{cccc}
\operatorname{Var}\left(M_{j k}\right) & 0 & \ldots & 0 \\
0 & \operatorname{Var}\left(M_{1}\right) & \ldots & \ldots \\
\ldots & \ldots & \ldots & 0 \\
0 & \ldots & 0 & \operatorname{Var}\left(M_{k-1}\right)
\end{array}\right) \times\left(\begin{array}{c}
\frac{\delta g}{\delta M_{j k}} \\
\frac{\delta g}{\delta M_{1}} \\
\ldots \\
\delta g \\
\delta M_{k-1}
\end{array}\right)
$$

So

$$
\operatorname{Var}\left(g\left(M_{j k}, M_{1}, \ldots, M_{k-1}\right)\right)=\left(\frac{\delta g}{\delta M_{j k}}\right)^{2} \operatorname{Var}\left(M_{j k}\right)+\sum_{a=1}^{k-1}\left(\frac{\delta g}{\delta M_{a}}\right)^{2} \operatorname{Var}\left(M_{a}\right)
$$

with

$$
\begin{aligned}
\frac{\delta g}{\delta M_{j k}} & =\left(\prod_{z=1}^{k-1}\left(1-\frac{M_{z}}{R_{z}}\right)\right) \times \frac{1}{R_{z}}=\frac{\widehat{S}\left(t_{k-11}\right) \widehat{\lambda}_{j}\left(t_{k}\right)}{M_{j k}} \text { et } \\
\frac{\delta g}{\delta M_{a}} & =\frac{M_{j k}}{R_{k}}\left(\prod_{z=1, z \neq a}^{k-1}\left(1-\frac{M_{z}}{R_{z}}\right)\right)\left(\frac{-1}{R_{a}}\right)=-\frac{\widehat{S}\left(t_{k-11}\right) \widehat{\lambda}_{j}\left(t_{k}\right)}{\left(R_{a}-M_{a}\right)} .
\end{aligned}
$$

We remark that $M_{j k}$ and $M_{k}$ follow the Binomial Law respectively. Indeed:

$$
\begin{aligned}
& M_{j k} \hookrightarrow \mathcal{B}\left(R_{k}, \frac{M_{j k}}{R_{k}}, R_{k} \times\left(\frac{M_{j k}}{R_{k}}\right) \times\left(1-\frac{M_{j k}}{R_{k}}\right)\right) \Longrightarrow \operatorname{Var}\left(M_{j k}\right)=R_{k} \times\left(\frac{M_{j k}}{R_{k}}\right) \times\left(1-\frac{M_{j k}}{R_{k}}\right)=\frac{M_{j k}\left(R_{k}-M_{k}\right)}{R_{k}} ; \\
& M_{k} \hookrightarrow \mathcal{B}\left(R_{k}, \frac{M_{k}}{R_{k}}, R_{k} \times\left(\frac{M_{k}}{R_{k}}\right) \times\left(1-\frac{M_{k}}{R_{k}}\right)\right) \Longrightarrow \operatorname{Var}\left(M_{k}\right)=R_{k} \times\left(\frac{M_{k}}{R_{k}}\right) \times\left(1-\frac{M_{k}}{R_{k}}\right)=\frac{M_{k}\left(R_{k}-M_{k}\right)}{R_{k}}
\end{aligned}
$$


Replacing respectively these quantities in (29), we obtain:

$$
\begin{aligned}
\operatorname{Var}\left(g\left(M_{j k}, M_{1}, \ldots, M_{k-1}\right)\right)= & \operatorname{Var}\left(\left(\widehat{S}\left(t_{k}\right) \times \widehat{\lambda}_{j}\left(t_{k}\right)\right)\right) \\
= & \left.\left(\prod_{z=1}^{k-1}\left(1-\frac{M_{z}}{R_{z}}\right)\right) \frac{1}{R_{k}}\right)^{2}\left(\frac{M_{j k}\left(R_{k}-M_{j k}\right)}{R_{k}}\right) \\
& \left.+\sum_{a=1}^{k-1}\left(\frac{M_{j k}}{R_{k}}\left(\prod_{z=1, z \neq a}\left(1-\frac{M_{z}}{R_{z}}\right)\right)\left(\frac{-1}{R_{a}}\right)\right)^{2}\left(\frac{M_{a}\left(R_{a}-M_{a}\right)}{R_{a}}\right)\right) \\
= & \left(\frac{\widehat{S}\left(t_{k-1}\right) \widehat{\lambda}_{j}\left(t_{k}\right)}{M_{j k}}\right)^{2}\left(\frac{M_{j k}\left(R_{k}-M_{j k}\right)}{R_{k}}\right) \\
& +\sum_{a=1}^{k-1}\left(-\frac{\widehat{S}\left(t_{k-1}\right) \widehat{\lambda}_{j}\left(t_{k}\right)}{R_{a}-M_{a}}\right)^{2}\left(\frac{M_{a}\left(R_{a}-M_{a}\right)}{R_{a}}\right) \\
= & \left(\widehat{S}\left(t_{k-1}\right) \widehat{\lambda}_{j}\left(t_{k}\right)\right)^{2}\left(\frac{R_{k}-M_{k}}{M_{j k} R_{k}}+\sum_{a=1}^{k-1}\left(\frac{M_{a}}{\left(R_{a}-M_{a}\right) R_{a}}\right)\right) .
\end{aligned}
$$

The relation (30) gives a first part of the expression (28).

The same procedure is used to determine an expression of

$$
\operatorname{Cov}\left(\left(\widehat{S}\left(t_{k-1}\right) \times \widehat{\lambda}_{j}\left(t_{k}\right)\right),\left(\widehat{S}\left(t_{b-1} \times \widehat{\lambda}\left(t_{b}\right)\right)\right) .\right.
$$

So, we pose

$$
g\left(M_{k j}, M_{1}, \ldots, M_{k-1}\right)=\left(\prod_{z=1}^{k-1}\left(1-\frac{M_{z}}{R_{z}}\right)\right) \frac{M_{j k}}{R_{k}}
$$

and

$$
f\left(M_{b j}, M_{1}, \ldots, M_{k-1}, M_{k}, \ldots, M_{b-1}\right)=\left(\prod_{z=1}^{b-1}\left(1-\frac{M_{z}}{R_{z}}\right)\right) \frac{M_{j b}}{R_{b}},
$$

with $k=1 \cdots\left(i^{\prime}-1\right)$ et $b=(k+1) \cdots i^{\prime}$.

As $\operatorname{Cov}\left(M_{j z}, M_{j z^{\prime}}\right)=0$ if $z \neq z^{\prime}$, so

$$
\operatorname{Cov}\left(M_{z}, M_{j Z^{\prime}}\right)=0 \text { si } z \neq z^{\prime} \text { et } \mathbb{C o v}\left(M_{z}, M_{z^{\prime}}\right)=0 \text { si } z \neq z^{\prime} .
$$

To finish, it remains to calculate the quantity:

$$
\begin{aligned}
& \operatorname{Cov}\left(g\left(M_{k j}, M_{1}, \ldots, M_{k-1}\right), f\left(M_{b j}, M_{1}, \ldots, M_{k-1}, M_{k}, \ldots, M_{b-1}\right)\right) \\
= & \left(\frac{\delta g}{\delta M_{j k}}\right)\left(\frac{\delta f}{\delta M_{k}}\right) \operatorname{Cov}\left(M_{j k}, M_{k}\right)+\sum_{a=1}^{k-1}\left[\left(\frac{\delta g}{\delta M_{a}}\right)\left(\frac{\delta f}{\delta M_{a}}\right) \operatorname{Var}\left(M_{a}\right)\right] .
\end{aligned}
$$

In deriving $g$ and $f$ respectively by $M_{j k}$ and $M_{k}$, we get:

$$
\frac{\delta g}{\delta M_{j k}}=\left(\prod_{z=1}^{k-1}\left(1-\frac{M_{z}}{R_{z}}\right)\right) \frac{1}{R_{z}}=\frac{\widehat{S}\left(t_{k-1}\right) \widehat{\lambda}_{j}\left(t_{k}\right)}{M_{j k}}
$$

and

$$
\frac{\delta f}{\delta M_{k}}=\frac{M_{j b}}{R_{b}}\left(\prod_{z=1, z \neq k}^{k-1}\left(1-\frac{M_{z}}{R_{z}}\right)\right)\left(\frac{-1}{R_{z}}\right)=\frac{\widehat{S}\left(t_{b-1}\right) \widehat{\lambda}_{j}\left(t_{b}\right)}{\left(1-\frac{M_{k}}{R_{k}}\right)}\left(-\frac{1}{R_{k}}\right)=\frac{\widehat{S}\left(t_{b-1}\right) \widehat{\lambda}_{j}\left(t_{b}\right)}{R_{k}-M_{k}} .
$$


The calculation of $\operatorname{Cov}\left(M_{j k}, M_{k}\right)$ gives:

$$
\begin{aligned}
\operatorname{Cov}\left(M_{j k}, M_{k}\right) & =\operatorname{Cov}\left(M_{j k}, \sum_{z=1}^{m} M_{z k}\right)=\sum_{z=1}^{m} \mathbb{C o v}\left(M_{j k}, M_{z k}\right)=\sum_{z=1, z \neq i}^{m} \mathbb{C o v}\left(M_{j k}, M_{z k}\right)+\operatorname{Var}\left(M_{j k}\right) \\
& =\frac{M_{j k}\left(R_{k}-M_{k}\right)}{R_{k}} .
\end{aligned}
$$

In deriving $g$ and $f$ by $M_{a}$ again, we obtain respectively:

$$
\begin{gathered}
\frac{\delta g}{\delta M_{a}}=\frac{M_{j k}}{R_{k}}\left(\prod_{z=1, z \neq a}^{k-1}\left(1-\frac{M_{z}}{R_{z}}\right)\right)\left(-\frac{1}{R_{z}}\right)=-\frac{\widehat{S}\left(t_{k-1}\right) \widehat{\lambda}_{j}\left(t_{k}\right)}{R_{a}-M_{a}} \\
\frac{\delta f}{\delta M_{a}}=\frac{M_{j b}}{R_{b}}\left(\prod_{z=1, z \neq k}^{b-1}\left(1-\frac{M_{z}}{R_{z}}\right)\right)\left(-\frac{1}{R_{a}}\right)=-\frac{\widehat{S}\left(t_{b-1}\right) \widehat{\lambda}_{j}\left(t_{b}\right)}{R_{a}-M_{a}} .
\end{gathered}
$$

Calculating the variance of $M_{a}$ gives:

$$
\operatorname{Var}\left(M_{a}\right)=\frac{M_{a}\left(R_{a}-M_{a}\right)}{R_{a}} .
$$

By replacing all the previous calculations in (31), we obtain:

$$
\begin{aligned}
& \operatorname{Cov}\left(g\left(M_{k j}, M_{1}, \ldots, M_{k-1}\right), f\left(M_{b j}, M_{1}, \ldots, M_{k-1}, M_{k}, \ldots, M_{b-1}\right)\right) \\
= & \operatorname{Cov}\left(\left(\widehat{S}\left(t_{k-1}\right) \times \widehat{\lambda}_{j}\left(t_{k}\right)\right),\left(\widehat{S}\left(t_{b-1}\right) \times \widehat{\lambda}_{j}\left(t_{b}\right)\right)\right) \\
= & \left(\frac{\widehat{S}\left(t_{k-1}\right) \widehat{\lambda}_{j}\left(t_{k}\right)}{M_{j k}}\right)\left(-\frac{\widehat{S}\left(t_{b-1}\right) \widehat{\lambda}_{j}\left(t_{b}\right)}{R_{a}-M_{a}}\right)\left(\frac{M_{j k}\left(R_{k}-M_{k}\right)}{R_{k}}\right) \\
& +\sum_{a=1}^{k-1}\left(-\frac{\widehat{S}\left(t_{k-1}\right) \widehat{\lambda}_{j}\left(t_{k}\right)}{R_{a}-M_{a}}\right)\left(-\frac{\widehat{S}\left(t_{b-1}\right) \widehat{\lambda}_{j}\left(t_{b}\right)}{R_{a}-M_{a}}\right)\left(\frac{M_{a}\left(R_{a}-M_{a}\right)}{R_{a}}\right) \\
= & \widehat{S}\left(t_{k-1}\right) \widehat{\lambda}_{j}\left(t_{k}\right) \times \widehat{S}\left(t_{b-1}\right) \widehat{\lambda}_{j}\left(t_{b}\right) \times\left(-\frac{1}{R_{k}}+\sum_{a=1}^{k-1} \frac{M_{a}}{\left(R_{a}-M_{a}\right) R_{a}}\right) .
\end{aligned}
$$

Finally, replacing (32) and (30) in (28), gives the formula of the variance of the sought estimator.

$$
\begin{aligned}
\operatorname{Var}\left(\hat{F}_{j}\left(t_{i}\right)\right)= & \operatorname{Var}\left(\sum_{t \leq t_{i}^{\prime}}\left(\widehat{S}(t) \times \widehat{\lambda}_{j}(t)\right)\right) \\
= & \sum_{k=1}^{i^{\prime}} \operatorname{Var}\left(\left(\widehat{S}(t) \times \widehat{\lambda}_{j}(t)\right)+2 \sum_{k=1}^{i^{\prime}-1} \sum_{b=k+1}^{i^{\prime}} \operatorname{Cov}\left(\left(\widehat{S}\left(t_{k}\right) \times \widehat{\lambda}\left(t_{k}\right)\right),\left(\widehat{S}\left(t_{b}\right) \times \widehat{\lambda}_{j}\left(t_{b}\right)\right)\right)\right. \\
= & \sum_{k=1}^{t^{\prime}}\left(\left(\widehat{S}\left(t_{k-1}\right) \widehat{\lambda}_{j}\left(t_{k}\right)\right)^{2}\right)\left(\frac{R_{k}-M_{k}}{M_{j k} R_{k}}+\sum_{a=1}^{k-1}\left(\frac{M_{a}}{\left(R_{a}-M_{a}\right) R_{a}}\right)\right) \\
& +2 \sum_{k=1}^{i^{\prime}-1} \sum_{b=k+1}^{i^{\prime}}\left(\widehat{S}\left(t_{k-1}\right) \widehat{\lambda}_{j}\left(t_{k}\right) \times \widehat{S}\left(t_{b-1}\right) \widehat{\lambda}_{j}\left(t_{b}\right)\right) \times\left(-\frac{1}{R_{k}}+\sum_{a=1}^{k-1} \frac{M_{a}}{\left(R_{a}-M_{a}\right) R_{a}}\right) .
\end{aligned}
$$

This completes the proof of the cumulative incidence variance estimator in the presence of competiting risks.

This ends the proof of the theorem.

Acknowledgments. The author sincerely thanks everyone who has reviewed this paper. A special thanks to the friends who corrected the English version of this paper. They are Dr. Safosso and Mrs. Esther Dankoni. 


\section{References}

1. O. O. Aalen, "Nonparametric estimation of partial transition probabilities in multiple decrement models," The Annals of Statistics, vol. 6, no. 3, pp. 534-545, 1978.

2. O. O. Aalen, "Nonparametric inference for a family of counting processes," Ann. Statist., vol. 6, no. 4, pp. 701-726, 1978.

3. P. K. Andersen, Ø. Borgan, R. D. Gill, and N. Keiding, "Statistical models based on counting Processes". Springer-Verlag, 1991.

4. P. K. Andersen, Ø. Borgan, R. D. Gill, and N. Keiding, "It's Statistical Models Based on Counting Processes", Springer-Verlag. 1992.

5. P.K. Andersen, Ø. Borgan, R.D. Gill, and N. Keiding, "Statistical Models based on Counting Processes," Springer Series in Statistics, Spring-Verlag, New York, 1993.

6. A. Belot, "Modélisation flexible des données de survie en présence de risques concurrents et apports de la méthode du taux en excès". Thèse de doctorat. Université de la Méditerranée. http://cybertim.timone.univmrs.fr/recherche/doc-recherche/statistiques/These-AurelienBelot/publication-file 2009.

7. P. M. Camblor, "Kaplan-Meier estimator in competing risk contexts", vol. 45, no. 4, pp. 1161-1173, Hacettepe Journal of Mathematics and Statistics, 2016.

8. C. Carbon, C. Huber, J. P. Lecoutre, "Analyse Statistiques des Durées de vie", Chr. Gouriéroux, ed. Droesbeke, Fichet, Tassi, Economica, 1989.

9. M. Crowder, "Classical competing risks", London: Chapman and Hall. 2001.

10. D. Commenges, "Risques compétitifs et modèles multi-états en épidémiologie", Revue d'Épidémiologie et Santé Publique, Elsevier Masson, vol. 47, no. 6, pp. 605-611, 1999.

11. C. Com-nougué, "Estimation des risques associés à des événements multiples", Revue d'Épidémiologie et de Santé Publique, Elsevier Masson, vol. 47, no. 1, pp. 75-85, 1999.

12. D. Cox, and D. Oakes, "Analysis of Survival Data", Chapman and Hall, London, 1984.

13. G. E. Dinse and M. G. Larson, "A note on semi-Markov models for partially censored data" Biometrika, vol. 73, no. 2, pp. 379-386, 1986.

14. T R fleming and D P. Harrington, "Counting processes and survival analysis", John Wiley and Sons, Inc., New York, 1991.

15. R. J. Gray, "A class of k-sample tests for comparing the cumulative incidence of a competing risk", The Annals of statistics, JSTOR, vol. 16, no. 3, pp. 1141-1154, 1988.

16. J. Heckman and B. Honoré, "The identifiability of the competing risks models", Biometrika, vol. 76, no. 2, pp. 325-330, 1989.

17. C. Hill, C. Com-Nougué, A. Kramar, T. Moreau, J. O’Quigley, and R. Senoussi, "Analyse statistique des données de survie", Cl. Chastang, Flammarion Sciences, 1996, 3ème édition, 2000.

18. C. Huber, "Analyse des durées de survie", http://www.biomedicale.parisdescartes.fr/survie/enseign/surviesansi.pdf.

19. J. Kalbfleisch and R. Prentice, "The Statistical Analysis of Failure Time Data", John Wiley, New York, 1980.

20. J. P. Klein, "Modelling competing risks in cancer studies", Statistics in Medicine, vol. 25, no. 6, pp. 1015-1034, 2006.

21. J. P. Klein and M. L. Moeschberger, "Survival Analysis : Techniques for Censored and Truncated Data", Second Edition. New York, Springer, 2003.

22. A. Latouche, "Modèles de Régression en Présence de Compétition", Thèse de Doctorat, Université de Paris, Paris,hal.archives-ouvertes.fr, 2004.

23. E. Marubini and M. G. Valsecchi, "Analysing survival data from clinical trials and observational studies", Statistics in Practice, New York, USA, 1995.

24. R. G. Miller, "Survival Analysis", John Wiley and Sons, Inc., New York, Edition, 1998.

25. D. A. N. Njamen and J. W. Ngatchou, "Nelson-Aalen and Kaplan-Meier estimators in eompeting Risks", Applied Mathematics, vol. 5, no. 4, pp. 765-776, 2014.

26. D. A. N. Njamen, "Convergence of the Nelson-Aalen estimator in competing risks", International Journal of Statistics and Probability, Canadian Center of Science and Education, vol. 6, no. 3, pp. 9-23, 2017.

27. E. Peña, R. Strawderman, and M. Hollander, "Nonparametric estimation with recurrent event data", Journal of the American Statistical Association, vol. 96, no. 456, pp. 1299Ü1315, 2001.

28. R. L. Prentice, "Linear rank tests with right-censored Data", Biometrika, vol. 65, pp. 167-179, 1978.

29. Saranya1 and Karthikeyan, "A Comparison study of Kaplan Meier and Nelson-Aalen methods in survival analysis", International Journal for Research in Emerging Science and Technology, vol. 2, no. 11, 2015.

30. R. S. Stocker IV and A. Adekpedjou, "Optimal goodness-of-fit tests for recurrent event data", Lifetime Data Anal, vol. 17, no. 3, pp. 409-432, 2011.

31. A. A. Tsiatis, "A nonidentifiability aspect of the problem of competing risks", Proc. Nat. Acad. Sci., USA. vol. 72 , no. 1, pp. 20-22, 1975. 